American Journal of Applied Sciences 6 (6): 1031-1035, 2009

ISSN 1546-9239

(C) 2009 Science Publications

\title{
Hyperspectral Imagery for Mapping Disease Infection in Oil Palm Plantation Using Vegetation Indices and Red Edge Techniques
}

\author{
Helmi Zulhaidi Mohd Shafri and Nasrulhapiza Hamdan \\ Department of Civil Engineering, Faculty of Engineering, \\ University Putra Malaysia, 43400 Serdang, Selangor, Malaysia
}

\begin{abstract}
Problem statement: Large scale plantation of oil palm trees requires on-time detection of diseases as the ganoderma basal stem rot disease was present in more than $50 \%$ of the oil palm plantations in Peninsular Malaysia. Approach: To deal with this problem, airborne hyperspectral imagery offers a better solution in order to detect and map the oil palm trees that were affected by the disease on time. Airborne hyperspectral can provide data on user requirement and has the capability of acquiring data in narrow and contiguous spectral bands which makes it possible to discriminate between healthy and diseased plants better compared to multispectral imagery. By using vegetation indices and red edge techniques, the condition of oil palm trees could be determined accurately. Results: Generally, all of these techniques showed better results as they could give accuracy between 73 and $84 \%$. The highest accuracy was achieved by using Lagrangian interpolation technique with $84 \%$ of overall accuracy. Conclusion/Recommendations: The red edge based techniques were more effective than vegetation indices in detecting Ganoderma-infected oil palm trees plantation since there were three out of four techniques that could yield high accuracy results.
\end{abstract}

Key words: Airborne sensor, oil palm, plant stress, vegetation indices, red edge

\section{INTRODUCTION}

Dealing with disease problem in oil palm plantation involves a variety of curative measures in which disease detection and mapping play a central role. Hyperspectral remote sensing data offer a better chance of disease detection compared to multispectral imagery ${ }^{[1]}$. This study implemented the advantage of using hyperspectral airborne imagery data in order to find the best technique in discriminating the healthy and stressed oil palm trees.

This study can be considered as an important research for oil palm industry especially in Malaysia since there is no existing published research on the use of airborne hyperspectral imagery to detect Ganodermainfected oil palm trees. Six vegetation indices and four red edge techniques were tested on hyperspectral image which were the Normalized Difference Vegetation Indices (NDVI), Renormalized Vegetation Index $(\mathrm{RDVI})^{[2]}$, Simple Ratio Index (SRI) ${ }^{[3]}$, Modified Simple Ratio (MSR) ${ }^{[4]}$, Soil Adjusted Vegetation Index $(\mathrm{SAVI})^{[5]}$, Optimized Soil-Adjusted Vegetation Index $(\mathrm{OSAVI})^{[6]}$, Lagrangian interpolation technique ${ }^{[7]}$, Vogelmann red edge $(\mathrm{VOG} 1)^{[8]}$, linear four point interpolation ${ }^{[9]}$ and maximum first derivative $\mathrm{e}^{[7]}$.
Vegetation indices are widely used for the estimation of crop and vegetation variables by using visible and Near Infrared Regions (NIR) of the electromagnetic spectrum. Healthy plant typically displays very low reflectance and transmittance in visible region and very high reflectance and transmittance in NIR. It was due to strong absorptance by photosynthetic and accessory plant pigment in visible region and little absorptance by sub cellular particles or pigments and also because there is considerable scattering at mesophyll cell wall interfaces in NIR region ${ }^{[10-13]}$. This sharp dissimilarity in reflectance properties between visible and NIR wavelengths underpins a majority of remote approaches for monitoring and managing crop and natural vegetation communities ${ }^{[14,15]}$. Thus the presence of stresses in oil palm trees will be associated with the chlorophyll absorption in reflectance and the normalized pigment chlorophyll vegetation indexes which will be showing a loss of chlorophyll pigment compared to healthy oil palm trees.

The other methods explored in this study were the red edge techniques. The red edge region which is located at the red-near infrared $(680$ and $780 \mathrm{~nm})$ transition in leaf reflectance has been shown to have

Corresponding Author: Helmi Zulhaidi M. Shafri, Department of Civil Engineering, Faculty of Engineering, University Putra Malaysia, 43400 Serdang, Selangor, Malaysia Tel: 03-89466459 Fax: 03-86567129 
high information content for vegetation spectra ${ }^{[16]}$. The abrupt change between 680 and $780 \mathrm{~nm}$ caused by the combined effects of strong absorption in the red wavelengths and high reflectance in the NIR wavelengths is due to leaf internal scattering ${ }^{[17]}$. A shifting in the red edge slope and wavelength of maximum slope towards a longer wavelength could be due to the increasing amount of chlorophyll, otherwise the red edge position will shift towards a shorter wavelengths ${ }^{[18,19]}$. This condition has been used as a means to estimate changes in foliar chlorophyll content and also as an indicator of vegetation stress ${ }^{[17,19-21]}$.

\section{MATERIALS AND METHODS}

The study area is located in Serdang, Selangor Malaysia and the image was acquired by using Advanced Imaging Spectrometer for Applications (AISA) sensor in the year 2007. Figure 1 shows the location of the study area. AISA airborne hyperspectral imaging spectrometer is capable of collecting data within a spectral range of $430-900 \mathrm{~nm}$. Although AISA is capable of collecting up to 286 spectral channels within this range, the data rate associated with the short integration times (sampling rates) required of the sensor in most operational/flight modes, limits the number of channels. The full spectral mode, however, is useful for acquiring 286 band spectral signatures of specific targets that can be used to generate pure end members as well as for band selection purposes.

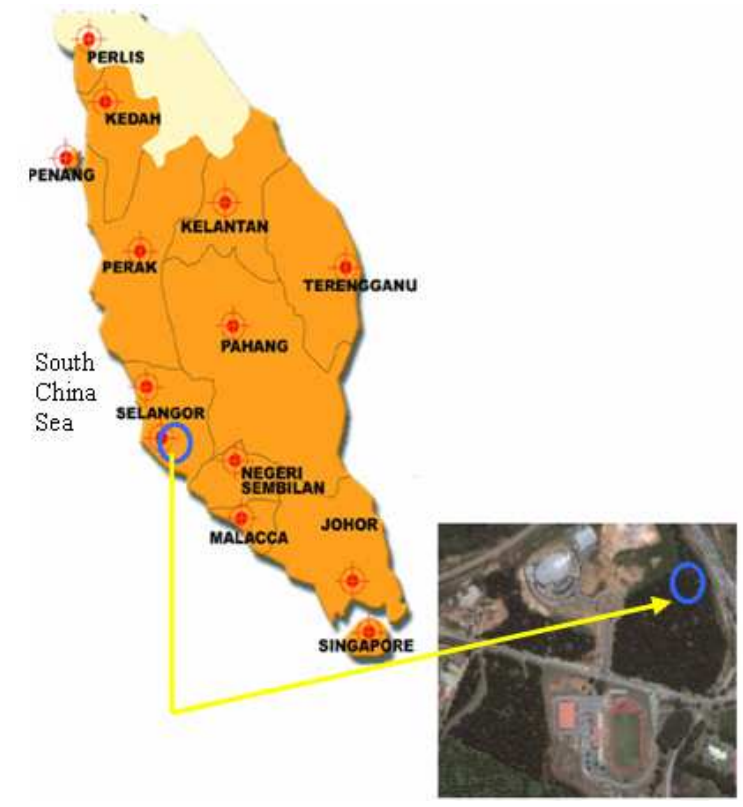

Fig. 1: Location of the study area in Selangor, Malaysia
Current operational collection configurations ranged from 10-70 spectral bands depending on the aircraft speed, altitude and the application. The spatial resolution of the data is $1 \mathrm{~m}$ with $1000 \mathrm{~m}$ flying altitude.

Generally, this study is comprised of two important parts. The first involved pre-processing and the second was processing. All these processes were implemented using ENVI 4.4 software. Figure 2 shows the overall flow of the steps that had been implemented in this study.

Pre-processing: Pre-processing involved several steps; geometric and radiometric correction, cross track illumination correction and Minimum Noise Fraction (MNF). Geometric and radiometric corrections were done in a separate software package under ENVI software environment called CaliGeo. After that, cross track illumination correction was implemented for removing variation in the image. Lastly, MNF transformation was performed on the hyperspectral image in order to determine the inherent dimensionality of the image data, to segregate noise in data and to reduce the computational requirement for subsequent processing. After the forward transformation, the MNF bands were inverse-transformed to obtain image data back into original (reflectance vs. wavelength) format but with reduced noise level.

Processing: Processing part involved calculation of vegetation indices and red edge position. For vegetation indices, it can be divided into two sub categories which are normalized difference and soil line vegetation indices.

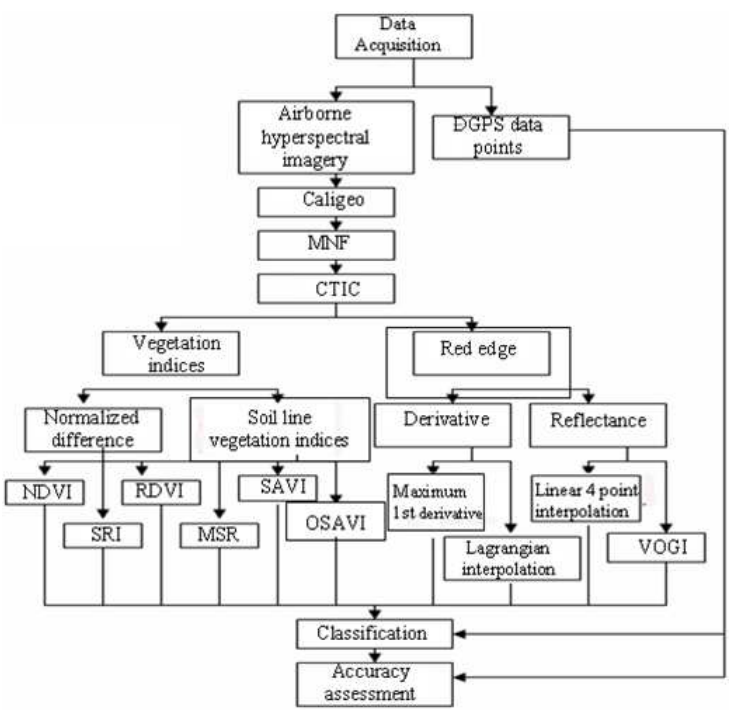

Fig. 2: Flowchart of methodology 
While for the red edge indices, the input data come from two types of data, in which the VOG1 and linear four points interpolation used reflectance data and maximum first derivative and Lagrangian interpolation techniques used first derivative data. Transformation from reflectance into first derivative reflectance image was performed using a derivative function. This function performs a numerical differentiation using three-point Lagrangian interpolation where the wavelengths of the respective AISA spectral bands are used as input for defining the spacing between the bands. All the calculations of the vegetation and red edge indices was done using 'Band Math' tool in ENVI 4.4 software. They were further used to classify healthy and stressed oil palm trees in the image. After that, accuracy assessment was performed on each of the classification result in order to identify the most suitable technique based on the highest overall accuracy. Confusion matrix was developed in order to calculate the overall accuracy for each classification result obtained from the vegetation indices and red edge techniques. 51 Differential Global Positioning System (DGPS) points measured using Trimble GeoXT for healthy and stressed oil palm trees collected from field work were overlaid to the classification results in order to measure the accuracy based on confusion matrix.

\section{RESULTS}

Table 1 shows the ranking from the highest to the lowest accuracy for both vegetation indices and red edge techniques. From Table 1, it can be seen that for vegetation indices, the highest accuracy was obtained by using MSR and SRI with $80 \%$ of overall accuracy for both of the techniques. RDVI, NDVI, SAVI and OSAVI get the same 78\% of overall accuracy. While for red edge techniques, the highest accuracy was obtained by using Lagrangian interpolation followed by VOG1, maximum 1st derivative and linear four point interpolation with the accuracies of $84,82,80$ and $73 \%$ respectively. Generally, three out of four techniques using red edge can give better discrimination of healthy and stressed oil palm trees since the accuracy was around 80 and $84 \%$. Figure 3 shows the example of healthy and stressed oil palm trees area using the Lagrangian interpolation technique. The healthy oil palm area was presented with green color class and the diseased oil palm area was presented by maroon color class. Other features such as roads and bare soils had been masked out from the image and they were presented as black color class.
Table 1: Accuracies for vegetation indices and red edge techniques ranked from the highest to the lowest accuracy

\begin{tabular}{ll}
\hline Techniques & $\begin{array}{l}\text { Overall accuracy } \\
\text { (correctly classified) }\end{array}$ \\
\hline Lagrangian interpolation technique & $84 \%$ \\
Vogelmann Red Edge Index (VOG1) & $82 \%$ \\
Maximum 1st derivative spectrum & $80 \%$ \\
Modified Simple Ratio (MSR) & $80 \%$ \\
Simple Ratio Index (SRI) & $80 \%$ \\
Renormalized Difference Vegetation Index (RDVI) & $78 \%$ \\
Normalized Difference Vegetation Index (NDVI) & $78 \%$ \\
Soil Adjusted Vegetation Index (SAVI) & $78 \%$ \\
Optimized Soil-Adjusted Vegetation Index (OSAVI) & $78 \%$ \\
Linear four point interpolation technique & $73 \%$ \\
\hline
\end{tabular}

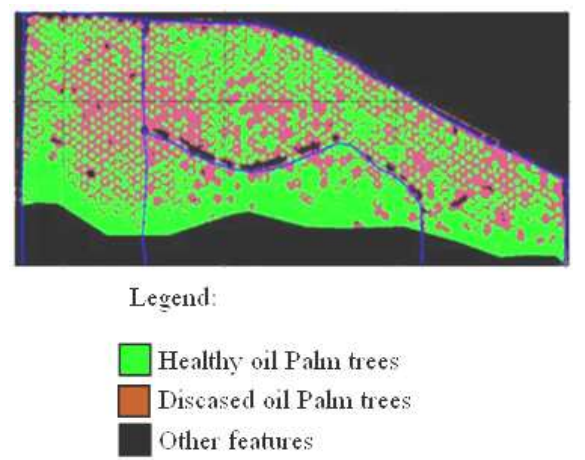

Fig. 3: The classification map of Lagrangian interpolation technique showing the locations of healthy and diseased oil palm trees detected from the airborne hyperspectral sensor

\section{DISCUSSION}

The classification for red edge based techniques was based on the wavelength value of the red edge peak between around $680-730 \mathrm{~nm}$. Using this technique, the pixels of healthy oil palm trees which contain more chlorophyll will have longer wavelength values compared to the diseased ones which will have shorter wavelength values. The obtained accuracy result from this technique was the highest compared to the other techniques. For the Lagrangian interpolation technique with the highest accuracy among other techniques, only eight points were misclassified for both healthy and stressed classes. Lagrangian interpolation used first derivative spectra as input for the calculation of the algorithm. In this algorithm, band 15, 16 and 17 that correspond to wavelength values of 715, 734 and 791 $\mathrm{nm}$ respectively of the sensor's data had been used in the calculation. The advantage of derivative spectroscopy is that it is relatively insensitive to variations in illumination intensity, which might be caused by changes in sun angle, cloud cover, topography and atmospheric attenuation. This could be the main reason for the success of this technique. 
This study further demonstrated the effectiveness of the Lagrangian interpolation technique for vegetation mapping as discussed for a case of a forest area in the United Kingdom ${ }^{[22]}$. However, as the use of hyperspectral remote sensing for detecting disease in oil palm plantation has never been implemented before, this study provides an important benchmark in evaluating the use of the technology and image processing algorithms.

For future studies, it is recommended to develop a more optimized index or technique specifically for detecting oil palm disease in order to further improve detection accuracy.

\section{CONCLUSION}

51 points for both healthy and diseased classes had been used to assess the classification results by using confusion matrix. The accuracies achievable by the techniques used can be ranked from the highest to lowest as Lagrangian interpolation (84\%), VOG1 $(82 \%)$, maximum $1^{\text {st }}$ derivative $(80 \%)$, MSR $(80 \%)$, SRI (80\%), RDVI (78\%), NDVI (78\%), SAVI (78\%), OSAVI (78\%) and linear four point interpolation (73\%). It shows that the Lagrangian interpolation technique would be able to discriminate between healthy and stressed oil palm trees accurately.

Other results that obtained higher accuracy were VOG1, Maximum first derivative, SRI and MSR. All these techniques achieved overall accuracy of $80 \%$ or more. It can be concluded that red edge based techniques are more effective than vegetation indices in detecting Ganoderma-infected oil palm trees. The finding from this study can be adopted by the oil palm plantation agencies for oil palm plantation management approach utilizing airborne hyperspectral imageries.

\section{ACKNOWLEDGEMENT}

The researchers would like to acknowledge the Ministry of Higher Education Malaysia in providing the grant for this research (FRGS 07-10-07-401FR).

\section{REFERENCES}

1. Franke, J. and G. Menz, 2007. Multi-temporal wheat disease detection by multi-spectral remote sensing. Precision Agric., 8: 161-172. DOI: 10.1007/s11119-007-9036-y

2. Rougean, J.L. and F.M. Breon, 1995. Estimating PAR absorbed by vegetation from bidirectional reflectance measurements. Remote Sens. Environ., 51: 375-84. DOI: 10.1016/0034-4257(94)00114-3
3. Jordan, C.F., 1976. Derivation of leaf area index from quality of light on the forest floor. Ecology, 50: 663-666. DOI: 10.2307/1936256

4. Chen, J., 1996. Evaluation of vegetation indices and modified simple ratio for boreal applications. Can. J. Remote Sens., 22: 229-242.

5. Huete, A.R., 1998. A Soil Adjusted Vegetation Index (SAVI). Remote Sens. Environ., 25: 295-309. DOI: 10.1016/0034-4257(88)90106-X)

6. Rondeaux, G., M. Steven and F. Baret, 1996. Optimization of soil-adjusted vegetation indices. Remote Sens. Environ., 55: 95-107. DOI: 10.1016/0034-4257(95)00186-7

7. Dawson, T.P. and P.J. Curran, 1998. A new technique for interpolating the reflectance red edge position. Int. J. Remote Sens., 19: 2133-2139. DOI: 10.1080/014311698214910

8. Vogelmann, J.E., B.N. Rock and D.M. Moss, 1993. Red edge spectral measurements from sugar maple leaves. Int. J. Remote Sens., 14: 1563-1575. DOI: 10.1080/01431169308953986

9. Guyot, G., F. Baret and S. Jacquemoud, 1992. Imaging Spectroscopy for Vegetation Studies. Imaging Spectroscopy: Fundamentals and Prospective Applications, pp: 145-165. http://www.ipgp.jussieu.fr/ jacquemoud/SJ_public ations.htm

10. Gausman, H.W., 1977. Reflectance of leaf components. Remote Sens. Environ., 6: 1-9. DOI: 10.1016/0034-4257(77)90015-3

11. Gausman, H.W., 1974. Leaf reflectance of nearinfrared radiation. Photogram. Eng. Remote Sens., 40: 183-191.

12. Chappelle, E.W., M.S. Kim and J.E. McMurtrey, 1992. Ratio Analysis of Reflectance Spectra (RARS)-An algorithm for the remote estimation of the concentrations of chlorophyll-a, chlorophyll-b and carotenoids in soybean leaves. Remote Sens. Environ., 39: 239-247. DOI: 10.1016/00344257(92)90089-3

13. Slaton, M.R., E.R. Hunt and W.K. Smith, 2001. Estimating near-infrared leaf reflectance from leaf structural characteristics. Am. J. Bot., 88: 278-284. DOI: $10.2307 / 2657019$

14. Knipling, E.B., 1970. Physical and physiological basis for the reflectance of visible and near-infrared radiation from vegetation. Remote Sens. Environ., 1: 155-159. DOI: 10.1016/S0034-4257(70)80021-9

15. Bauer, M.E., 1975. The role of remote sensing in determining the distribution and yield of crops. Adv. Agron., 27: 271-304. DOI: 10.1016/S00652113(08)70012-9 
16. Collins, W., 1978. Remote sensing of crop type and maturity. Photogram. Eng. Remote Sens., 44: 43-55. http://www.asprs.org/publications/pers/scans/1978j ournal/jan/1978_jan_43-55.pdf

17. Horler, D.N.H., J. Barber and A.R. Barringer, 1983. The red edge of plant leaf reflectance. Int. J. Remote Sens., 4: 273-288. DOI: $10.1080 / 01431168308948546$

18. Buschmann, C. and E. Nagel, 1993. In vivo spectroscopy and internal optics of leaves as basis for remote sensing of vegetation. Int. J. Remote Sens., 14: 711-722. DOI: 10.1080/01431169308904370

19. Clevers, J.G.P.W., S.M. De Jong, G.F. Epema, F. Van der Meer, W.H. Bakker and A.K. Skidmore, 2002. Derivation of the red edge index using MERIS standard band setting. Int. J. Remote Sens., 23: 3169-3184. DOI: 10.1080/01431160110104647
20. Curran, P.J., W.R. Windham and H.L. Gholz, 1995. Exploring the relationship between reflectance red edge and chlorophyll concentration in slash pine leaves. Tree Physiol., 15: 203-206. http://heronpublishing.com/tree/pdf/volume15/15203.pdf

21. Smith, K.L., M.D. Steven and J.J. Colls, 2004. Use of hyperspectral derivative ratios in the red edge region to identify plant stress responses to gas leak. Remote Sens. Environ., 92: 207-217. DOI:10.1016/j.rse.2004.06.002

22. Shafri, H.Z.M., M.A.M. Salleh and A. Ghiyamat, 2006. Hyperspectral remote sensing of vegetation using red edge position techniques. Am. J. Applied Sci., 3: 1864-1871.

http://www.scipub.org/fulltext/ajas/ajas3618641871.pdf 